Proyecciones Journal of Mathematics

Vol. 35, No 2, pp. 177-186, June 2016.

Universidad Católica del Norte

Antofagasta - Chile

\title{
Vertex equitable labeling of union of cyclic snake related graphs
}

\author{
P. Jeyanthi \\ Govindammal Aditanar College for Women, India \\ A. Maheswari \\ Kamaraj College of Engineering and Technology, India \\ and \\ M. Vijayalakshmi \\ Dr. G. U. Pope College of Engineering, India \\ Received : May 2015. Accepted : March 2016
}

\begin{abstract}
Let $G$ be a graph with $p$ vertices and $q$ edges and $A=\{0,1,2, \ldots$, $\left.\left\lceil\frac{q}{2}\right\rceil\right\}$. A vertex labeling $f: V(G) \rightarrow A$ induces an edge labeling $f^{*}$ defined by $f^{*}(u v)=f(u)+f(v)$ for all edges uv. For $a \in A$, let $v_{f}(a)$ be the number of vertices $v$ with $f(v)=a$. A graph $G$ is said to be vertex equitable if there exists a vertex labeling $f$ such that for all $a$ and $b$ in $A,\left|v_{f}(a)-v_{f}(b)\right| \leq 1$ and the induced edge labels are $1,2,3, \ldots, q$. In this paper, we prove that key graph $K Y(m, n), P\left(2 . Q S_{n}\right), P\left(m \cdot Q S_{n}\right)$, $C\left(n . Q S_{m}\right), N Q(m)$ and $K_{1, n} \times P_{2}$ are vertex equitable graphs.
\end{abstract}

Keywords : Vertex equitable labeling, vertex equitable graph, comb graph, key graph, path union graph, quadrilateral snake graph.

AMS Subject Classification : $05 C^{r} 78$. 


\section{Introduction}

All graphs considered here are simple, finite, connected and undirected. We follow the basic notations and terminology of graph theory as in [1]. A graph labeling is an assignment of integers to the vertices or edges or both, subject to certain conditions. There are several types of labeling and a detailed survey of graph labeling can be found in [2]. The vertex set and the edge set of a graph are denoted by $V(G)$ and $E(G)$ respectively. The concept of vertex equitable labeling was due to Lourdusamy and Seenivasan in [3] and further studied in [4]-[10]. Let $G$ be a graph with $p$ vertices and $q$ edges and $A=\left\{0,1,2, \ldots,\left\lceil\frac{q}{2}\right\rceil\right\}$. A graph $G$ is said to be vertex equitable if there exists a vertex labeling $f: V(G) \rightarrow A$ induces an edge labeling $f^{*}$ defined by $f^{*}(u v)=f(u)+f(v)$ for all edges $u v$ such that for all $a$ and $b$ in $A,\left|v_{f}(a)-v_{f}(b)\right| \leq 1$ and the induced edge labels are $1,2,3, \ldots, q$, where $v_{f}(a)$ be the number of vertices $v$ with $f(v)=a$ for $a \in A$. The vertex labeling $f$ is known as vertex equitable labeling. A graph $G$ is said to be a vertex equitable if it admits vertex equitable labeling. In this paper, we extend our study on vertex equitable labeling and prove that key graph $K Y(m, n), P\left(2 . Q S_{n}\right), P\left(m \cdot Q S_{n}\right)$, $C\left(n . Q S_{m}\right), N Q(m)$ and $K_{1, n} \times P_{2}$ are vertex equitable graphs. In [3], it is proved that the comb graph $P_{n} \odot K_{1}$ is a vertex equitable graph. In the following theorem we give an another vertex equitable labeling for the same graph $P_{n} \odot K_{1}$.

Theorem 1.1. The comb graph $P_{n} \odot K_{1}$ is a vertex equitable graph.

Proof. Let $V\left(P_{n} \odot K_{1}\right)=\left\{u_{i}, v_{i}: 1 \leq i \leq n\right\}$ and $E\left(P_{n} \odot K_{1}\right)=$ $\left\{u_{i} v_{i}: 1 \leq i \leq n\right\} \cup\left\{u_{i} u_{i+1}: 1 \leq i \leq n-1\right\}$. Here $\left|V\left(P_{n} \odot K_{1}\right)\right|=2 n$ and $E\left(P_{n} \odot K_{1}\right) \mid=2 n-1$. Let $A=\left\{0,1,2, \ldots,\left\lceil\frac{2 n-1}{2}\right\rceil\right\}$.

Define a vertex labeling $f: V\left(P_{n} \odot K_{1}\right) \rightarrow A$ as follows:

Case (i). When $n$ is even.

$f\left(u_{2 i-1}\right)=2(i-1), f\left(u_{2 i}\right)=2 i, f\left(v_{2 i-1}\right)=f\left(v_{2 i}\right)=2 i-1$ if $1 \leq i \leq \frac{n}{2}$.

Case (ii). When $n$ is odd.

$f\left(u_{2 i-1}\right)=2 i-1, f\left(v_{2 i-1}\right)=2(i-1)$ if $1 \leq i \leq\left\lceil\frac{n}{2}\right\rceil, f\left(v_{2 i}\right)=2 i, f\left(u_{2 i}\right)=$ $2 i-1$ if $1 \leq i \leq\left\lfloor\frac{n}{2}\right\rfloor$. It can be verified that the induced edge labels of $P_{n} \odot K_{1}$ are $1,2, \ldots, 2 n-1$ and $\left|v_{f}(a)-v_{f}(b)\right| \leq 1$ for all $a, b \in A$. Hence $P_{n} \odot K_{1}$ is a vertex equitable graph.

We use the following theorem and definitions in the subsequent section. 
Theorem 1.2. [3] The cycle $C_{n}$ is a vertex equitable graph if and only if $n \equiv 0 \operatorname{or} 3(\bmod 4)$.

Theorem 1.3. [7] The $k C_{4}$-snake is a vertex equitable graph.

Theorem 1.4. [4] Let $G_{1}\left(p_{1}, 2 n+1\right)$ and $G_{2}\left(p_{2}, q_{2}\right)$ be any two vertex equitable graphs with equitable labeling $f$ and $g$ respectively. Let $u$ and $v$ be the vertices of $G_{1}$ and $G_{2}$ respectively such that $f(u)=n+1$ and $g(v)=0$. Then the graph $G$ obtained by joining $u$ and $v$ by an edge is a vertex equitable graph.

Theorem 1.5. [9] Let $G_{1}\left(p_{1}, q\right), G_{2}\left(p_{2}, q\right), \ldots, G_{m}\left(p_{m}, q\right)$ be the vertex equitable graphs with $q$ is odd and $u_{i}, v_{i}$ be the vertices of $G_{i}(1 \leq i \leq m)$ labeled by 0 and $\left\lceil\frac{q}{2}\right\rceil$. Then the graph $G$ obtained by joining $v_{1}$ with $u_{2}$ and $v_{2}$ with $u_{3}$ and $v_{3}$ with $u_{4}$ and so on until we join $v_{m-1}$ with $u_{m}$ by an edge is also a vertex equitable graph.

Definition 1.6. Let $N Q(m)$ be the $n^{\text {th }}$ quadrilateral snake obtained from the path $u_{1}, u_{2}, \ldots, u_{m}$ by joining $u_{i}, u_{i+1}$ with $2 n$ new vertices $v_{j}^{i}$ and $w_{j}^{i}, 1 \leq i \leq m-1,1 \leq j \leq n$.

Definition 1.7. The key graph is a graph obtained from $K_{2}$ by appending one vertex of $C_{m}$ to one end point and comb graph $P_{n} \odot K_{1}$ to the other end of $K_{2}$. It is denoted as $K Y(m, n)$.

Definition 1.8. [11] Let $G_{1}, G_{2}, \ldots, G_{n}, n \geq 2$ be $n$ graphs and $u_{i}$ be a vertex of $G_{i}$ for $1 \leq i \leq n$. The graph obtained by adding an edge between $u_{i}$ and $u_{i+1}$ for $1 \leq i \leq n-1$ is called a path union of $G_{1}, G_{2}, \ldots, G_{n}$ and is denoted by $P\left(G_{1}, G_{2}, \ldots, G_{n}\right)$. When all the $n$ graphs are isomorphic to a graph $G$, it is denoted by $P(n \cdot G)$.

Definition 1.9. Let $G_{1}, G_{2}, \ldots, G_{n}$, be $n$ graphs and $u_{i}$ be a vertex of $G_{i}$ for $1 \leq i \leq n$. The graph obtained by adding an edge between $u_{i}$ and $u_{i+1}(1 \leq i \leq n-1), u_{n}$ and $u_{1}$ is called a cycle union of $G_{1}, G_{2}, \ldots, G_{n}$ and is denoted by $C\left(G_{1}, G_{2}, \ldots, G_{n}\right)$. When all the $n$ graphs are isomorphic to a graph $G$, it is denoted by $C(n . G)$.

\section{Main Results}

Theorem 2.1. The key graph $K Y(m, n)$ is a vertex equitable graph if $m \equiv 0$ or $3(\bmod 4)$. 


\section{Proof.}

Case(i). $\quad m \equiv 3(\bmod 4)$.

Let $G_{1}=C_{m}, G_{2}=P_{n} \odot K_{1}$. Since $G_{1}$ has $m$ edges and $G_{2}$ has $2 n-1$ edges, By Theorem 1.1 and Theorem 1.2, $P_{n} \odot K_{1}, C_{m}$ are vertex equitable graphs. Hence, by Theorem 1.4, $K Y(m, n)$ is a vertex equitable graph.

Case(ii). $m \equiv 0(\bmod 4)$.

Let $G_{1}=P_{n} \odot K_{1}, G_{2}=C_{m}$. Since $G_{1}$ has $2 n-1$ edges and $G_{2}$ has $m$ edges, By Theorem 1.1 and Theorem $1.2 P_{n} \odot K_{1}$ and $C_{n}$ are vertex equitable graphs. Hence by Theorem 1.4, $K Y(m, n)$ is a vertex equitable graph.

An example for the vertex equitable labeling of $K Y(7,5)$ is shown in Figure 1.

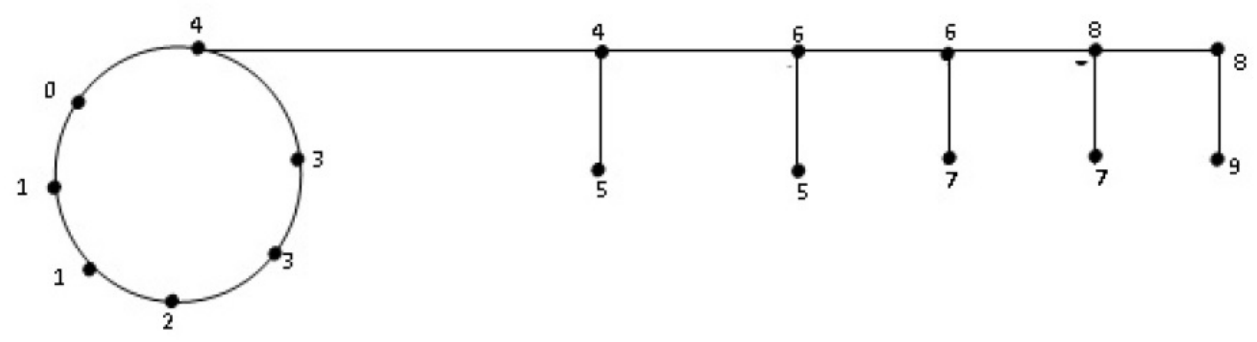

Figure 1

Theorem 2.2. The path union graph $P\left(2 . Q S_{n}\right)$ is a vertex equitable graph.

Proof. Let $V\left(P\left(2 . Q S_{n}\right)\right)=\left\{u_{i}, v_{i j}, w_{i j}: 1 \leq i \leq 2,1 \leq j \leq n\right\}$ and $E\left(P\left(2 . Q S_{n}\right)\right)=\left\{u_{1} u_{2}, u_{i} v_{i 1}, u_{i} w_{i 1}: 1 \leq i \leq 2\right\} \cup\left\{u_{i j} v_{i j}, u_{i j} w_{i j}: 1 \leq\right.$ $i \leq 2,1 \leq j \leq n\} \cup\left\{u_{i j} v_{i j+1}, u_{i j} w_{i j+1}: 1 \leq i \leq 2,1 \leq j \leq n-1\right\}$. Here $\left|V\left(P\left(2 . Q S_{n}\right)\right)\right|=6 n+2$ and $\left|E\left(P\left(2 . Q S_{n}\right)\right)\right|=8 n+1$. Let $A=$ $\left\{0,1,2, \ldots,\left\lceil\frac{8 n+1}{2}\right\rceil\right\}$.

Define a vertex labeling $f: V\left(\left(P\left(2 . Q S_{n}\right)\right)\right) \rightarrow A$ as follows: $f\left(u_{1}\right)=0, f\left(u_{2}\right)=\left\lceil\frac{8 n+1}{2}\right\rceil$. 
For $1 \leq j \leq n, f\left(u_{1 j}\right)=f\left(w_{1 j}\right)=2 j, f\left(v_{1 j}\right)=2 j-1, f\left(v_{2 j}\right)=f\left(v_{2 j}\right)=$ $f\left(u_{2 j}\right)=\left\lceil\frac{8 n+1}{2}\right\rceil-2 j, f\left(w_{2 j}\right)=\left\lceil\frac{8 n+1}{2}\right\rceil-2 j+1$.

It can be verifed that the induced edge labels of $P\left(2 . Q S_{n}\right)$ are $1,2, \ldots, 8 n+$ 1 and $\left|v_{f}(a)-v_{f}(b)\right| \leq 1$ for all $a, b \in A$. Hence $P\left(2 . Q S_{n}\right)$ is a vertex equitable graph.

Theorem 2.3. The path union graph $P\left(m \cdot Q S_{n}\right)$ is a vertex equitable graph if $m>2$.

Proof. Here $V\left|P\left(m \cdot Q S_{n}\right)\right|=m(3 n+1)$ and $E\left|P\left(m \cdot Q S_{n}\right)\right|=4 m n+$ $m-1$.

Case(i). $m$ is even.

Let $G_{i}=P\left(2 . Q S_{n}\right)$ for $1 \leq i \leq \frac{m}{2}$. By Theorem 2.2, $P\left(2 . Q S_{n}\right)$ is a vertex equitable graph. Since each $G_{i}$ has $8 n+1$ edges, by Theorem 1.5, $P\left(m . Q S_{n}\right)$ admits vertex equitable labeling if $m$ is even.

Case(ii). $\quad m$ is odd and take $m=2 k+1$.

By Case (i) $P\left(2 k \cdot Q S_{n}\right)$ is a vertex equitable graph. By Theorem 1.3, $n C_{4}$ snake is a vertex equitable graph. Let $G_{1}=P\left(2 k \cdot Q S_{n}\right)$ and $G_{2}=n C 4$. Since $G_{1}$ has $8 m n+2 m-1$ edges, by Theorem 1.4, $P\left(2 m+1 . Q S_{n}\right)$ admits vertex equitable labeling.

An example for the vertex equitable labeling of the graph obtained by the path union of 4 copies of $3 C_{4}$-snake is shown in Figure 2.

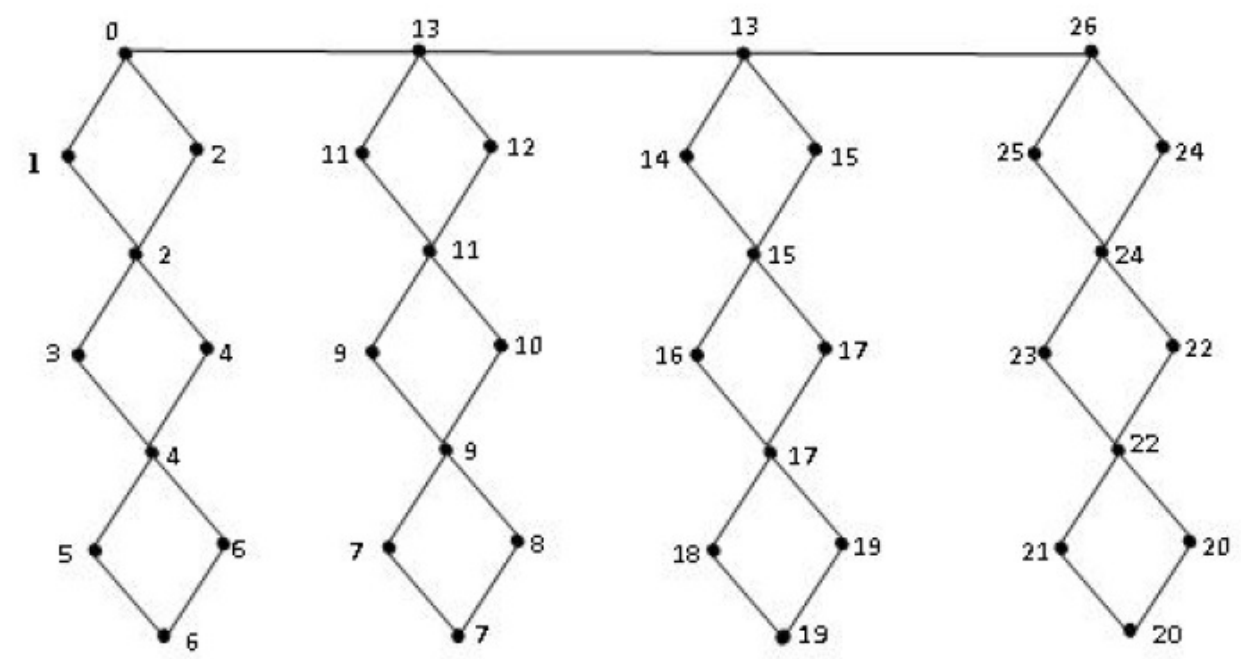

Figure 2 
Theorem 2.4. The graph obtained by the cycle union of $n$ copies of $m C_{4}$ snake, $C\left(n . Q S_{m}\right)$ is a vertex equitable graph if $n \equiv 0,3(\bmod 4)$.

Proof. Let $V\left(C\left(n . Q S_{m}\right)\right)=\left\{u_{i}, u_{i j}, v_{i j}, w_{i j}: 1 \leq i \leq n, 1 \leq j \leq m\right\}$ and $E\left(C\left(n \cdot Q S_{m}\right)\right)=\left\{u_{i} u_{i+1}: 1 \leq i \leq n-1\right\} \cup\left\{u_{n} u_{1}\right\} \cup\left\{u_{i} v_{i 1}, u w_{i 1}: 1 \leq\right.$ $i \leq n\} \cup\left\{u_{i j} v_{i j}, u_{i j} w_{i j}: 1 \leq i \leq n, 1 \leq j \leq m\right\} \cup\left\{u_{i j} v_{i j+1}, u_{i j} w_{i j+1}: 1 \leq i \leq\right.$ $n, 1 \leq j \leq m-1\}$. Here $\left|V\left(C\left(n \cdot Q S_{m}\right)\right)\right|=3 m n+n$ and $\left|E\left(C\left(n \cdot Q S_{m}\right)\right)\right|=$ $4 m n+n$. Let $A=\left\{0,1,2, \ldots,\left\lceil\frac{4 m n+n}{2}\right\rceil\right\}$.

Define a vertex labeling $f: V\left(C\left(n \cdot Q S_{m}\right)\right) \rightarrow A$ as follows:

Case (i). $n \equiv 0(\bmod 4)$.

$\mathrm{f}\left(\mathrm{u}_{2 i}\right)=(4 m+1) i$ if $1 \leq i \leq \frac{n}{2}$,

$f\left(u_{2 i-1}\right)= \begin{cases}(4 m+1)(i-1) & \text { if } 1 \leq i \leq \frac{n}{4} \\ (4 m+1)(i-1)+1 & \text { if } \frac{n}{4}+1 \leq i \leq \frac{n}{2},\end{cases}$

For $1 \leq j \leq m$,

$\mathrm{f}\left(\mathrm{v}_{(2 i-1) j}\right)= \begin{cases}(4 m+1)(i-1)+2 j & \text { if } 1 \leq i \leq \frac{n}{4} \\ (4 m+1)(i-1)+(2 j-1) & \text { if } \frac{n}{4}+1 \leq i \leq \frac{n}{2},\end{cases}$

$f\left(v_{2 i) j}\right)=(4 m+1) i-1-2(j-1)$ if $1 \leq i \leq \frac{n}{2}$,

$f\left(w_{(2 i-1) j}\right)= \begin{cases}(4 m+1)(i-1)+2 j-1 & \text { if } 1 \leq i \leq \frac{n}{4} \\ (4 m+1)(i-1)+2 j & \text { if } \frac{n}{4}+1 \leq i \leq \frac{n}{2}\end{cases}$

$f\left(w_{(2 i) j}\right)= \begin{cases}(4 m+1) i-2 j & \text { if } 1 \leq i \leq \frac{n}{4} \\ (4 m+1) i-2(j-1) & \text { if } \frac{n}{4}+1 \leq i \leq \frac{n}{2},\end{cases}$

$f\left(u_{(2 i-1) j}\right)= \begin{cases}(4 m+1)(i-1)+2 j & \text { if } 1 \leq i \leq \frac{n}{4} \\ (4 m+1)(i-1)+1+2 j & \text { if } \frac{n}{4}+1 \leq i \leq \frac{n}{2},\end{cases}$

$f\left(u_{(2 i) j}\right)=(4 m+1) i-2 j$ if $1 \leq i \leq \frac{n}{2}$.

Case (ii). $n \equiv 3(\bmod 4)$.

$\mathrm{f}\left(\mathrm{u}_{2 i}\right)=(4 m+1)(i-1)+(2 m+1)$ if $1 \leq i \leq\left\lfloor\frac{n}{2}\right\rfloor$,

$f\left(u_{2 i-1}\right)=\begin{array}{ll}(4 m+1)(i-1)+2 m & \text { if } 1 \leq i \leq\left\lceil\frac{n}{4}\right\rceil \\ (4 m+1)(i-1)+(2 m+1) & \text { if }\left\lceil\frac{n}{4}\right\rceil+1 \leq i \leq\left\lceil\frac{n}{2}\right\rceil,\end{array}$

For $1 \leq j \leq m$,

$f\left(u_{(2 i) j}\right)=(4 m+1)(i-1)+(2 m+1)+2 j$ if $1 \leq i \leq\left\lfloor\frac{n}{2}\right\rfloor$,

$\mathrm{f}\left(\mathrm{u}_{(2 i-1) j}\right)= \begin{cases}(4 m+1)(i-1)+2 m-2 j & \text { if } 1 \leq i \leq\left\lceil\frac{n}{4}\right\rceil \\ (4 m+1)(i-1)+2 m-2 j+1 & \text { if }\left\lceil\frac{n}{4}\right\rceil+1 \leq i \leq\left\lceil\frac{n}{2}\right\rceil,\end{cases}$

$f\left(v_{(2 i-1) j}\right)= \begin{cases}(4 m+1)(i-1)+2 m-2(j-1) & \text { if } 1 \leq i \leq\left\lceil\frac{n}{4}\right\rceil \\ (4 m+1)(i-1)+2 m+1-2 j & \text { if }\left\lceil\frac{n}{4}\right\rceil+1 \leq i \leq\left\lceil\frac{n}{2}\right\rceil,\end{cases}$

$f\left(v_{(2 i) j}\right)= \begin{cases}(4 m+1)(i-1)+2 m+2 j-1 & \text { if } 1 \leq i \leq\left\lceil\frac{n}{4}\right\rceil \\ (4 m+1)(i-1)+2 m+2 j & \text { if }\left\lceil\frac{n}{4}\right\rceil+1 \leq i \leq\left\lfloor\frac{n}{2}\right\rfloor,\end{cases}$ 


$$
\begin{aligned}
& f\left(w_{2 i-1) j}\right)= \begin{cases}(4 m+1)(i-1)+2 m-2 j+1 & \text { if } 1 \leq i \leq\left\lceil\frac{n}{4}\right\rceil \\
(4 m+1)(i-1)+2 m-2(j-1) & \text { if }\left\lceil\frac{n}{4}\right\rceil+1 \leq i \leq\left\lceil\frac{n}{2}\right\rceil,\end{cases} \\
& f\left(w_{(2 i) j}\right)= \begin{cases}(4 m+1)(i-1)+2 m+2 j & \text { if } 1 \leq i \leq\left\lceil\frac{n}{4}\right\rceil \\
(4 m+1)(i-1)+2 m+2 j+1 & \text { if }\left\lceil\frac{n}{4}\right\rceil+1 \leq i \leq\left\lfloor\frac{n}{2}\right\rfloor .\end{cases}
\end{aligned}
$$

It can be verified tha the induced edge labels of $C\left(n . Q S_{m}\right)$ are $1,2, \ldots, 4 m n+$ $n$ and $\left|v_{f}(a)-v_{f}(b)\right| \leq 1$ for all $a, b \in A$. Hence $C\left(n \cdot Q S_{m}\right)$ is a vertex equitable graph.

An example for the vertex equitable labeling of the graph obtained by the cycle union of 7 copies of $2 C_{4}$-snake is shown in Figure 3.

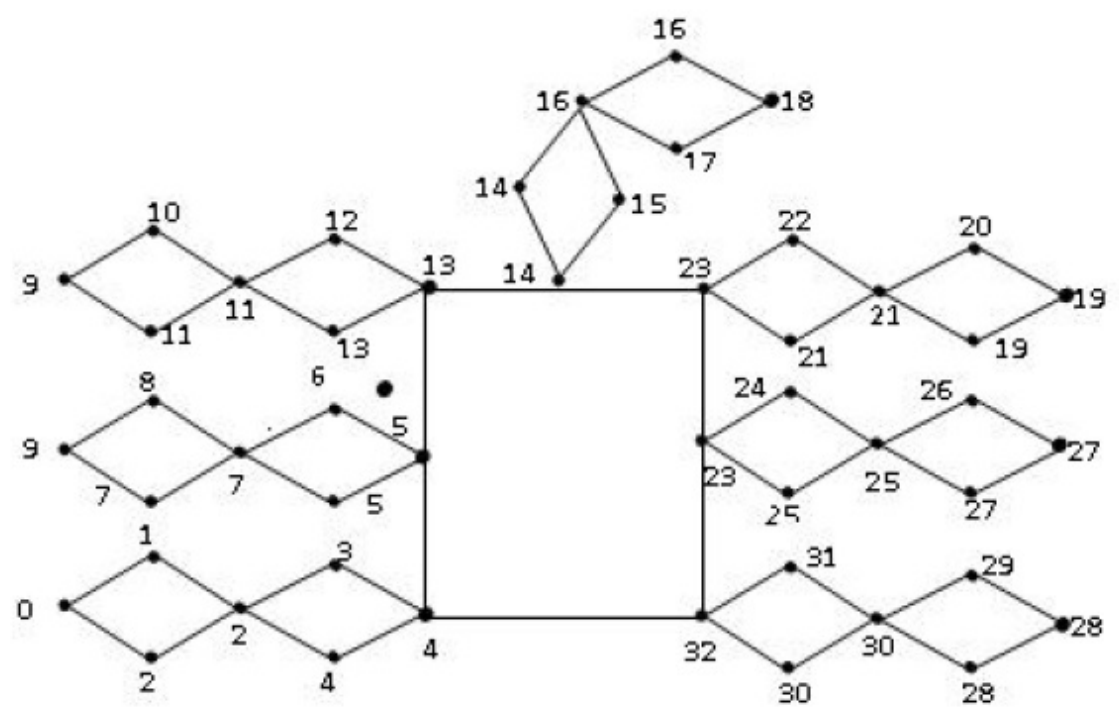

Figure 3

Theorem 2.5. The $n^{\text {th }}$ quadrilateral snake $N Q(m)$ is a vertex equitable graph if $n \geq 2$ is even. 
Proof. Let $V(N Q(m))=\left\{u_{i} / 1 \leq i \leq m\right\} \cup\left\{v_{j}^{i} / 1 \leq i \leq m-1,1 \leq\right.$ $j \leq n\} \cup\left\{w_{j}^{i} / 2 \leq i \leq m, 1 \leq j \leq n\right\}, E(N Q(m))=\left\{u_{i} u_{i+1} / 1 \leq i \leq\right.$ $n-1\} \cup\left\{u_{i} v_{j}^{i} / 1 \leq i \leq m-1,1 \leq j \leq n\right\} \cup\left\{u_{i} w_{j}^{i} / 2 \leq i \leq m, 1 \leq j \leq n\right\}$. Clearly $N Q(m)$ has $2(m-1) n+m$ vertices and $3(m-1) n+m-1$ edges. Let $A=\left\{0,1,2, \ldots,\left\lceil\frac{3 n(m-1)+m-1}{2}\right\rceil\right\}$.

Define a vertex labeling $f: V(N Q(m)) \rightarrow A$ as follows:

For $1 \leq i \leq m, f\left(u_{i}\right)=\left\lceil\frac{(3 n+1)(i-1)}{2}\right\rceil$,

For $1 \leq i \leq\left\lfloor\frac{m}{2}\right\rfloor, 1 \leq j \leq n, f\left(v_{j}^{2 i-1}\right)=(3 n+1)(i-1)+j$,

$f\left(v_{j}^{2 i}\right)=(3 n+1)(i-1)+\left\lceil\frac{(3 n+1)}{2}\right\rceil+(j-1)$.

For $1 \leq i \leq\left\lfloor\frac{m}{2}\right\rfloor, 1 \leq j \leq \frac{n}{2}, f\left(w_{j}^{2 i-1}\right)=(3 n+1)(i-1)+\left\lceil\frac{(3 n+1)}{2}\right\rceil-2 j$, $f\left(w_{\frac{n}{2}+j}^{2 i-1}\right)=(3 n+1)(i-1)+\left\lceil\frac{(3 n+1)}{2}\right\rceil-(2 j-1)$.

For $1 \leq i \leq\left\lfloor\frac{m}{2}\right\rfloor, 1 \leq j \leq \frac{n}{2}, f\left(w_{j}^{2 i}\right)=(3 n+1) i-(2 j-1)$, $f\left(w_{\frac{n}{2}+j}^{2 i}\right)=(3 n+1) i-(2 j-2)$.

It can be verified that the induced edge labels of $N Q(m)$ are $1,2, \ldots, 3(m-$ 1) $n+m-1$ and $\left|v_{f}(a)-v_{f}(b)\right| \leq 1$ for all $a, b \in A$. Hence $N Q(m)$ is a vertex equitable graph.

An example for the vertex equitable labeling of $4 Q(4)$ is shown in Figure 4 .

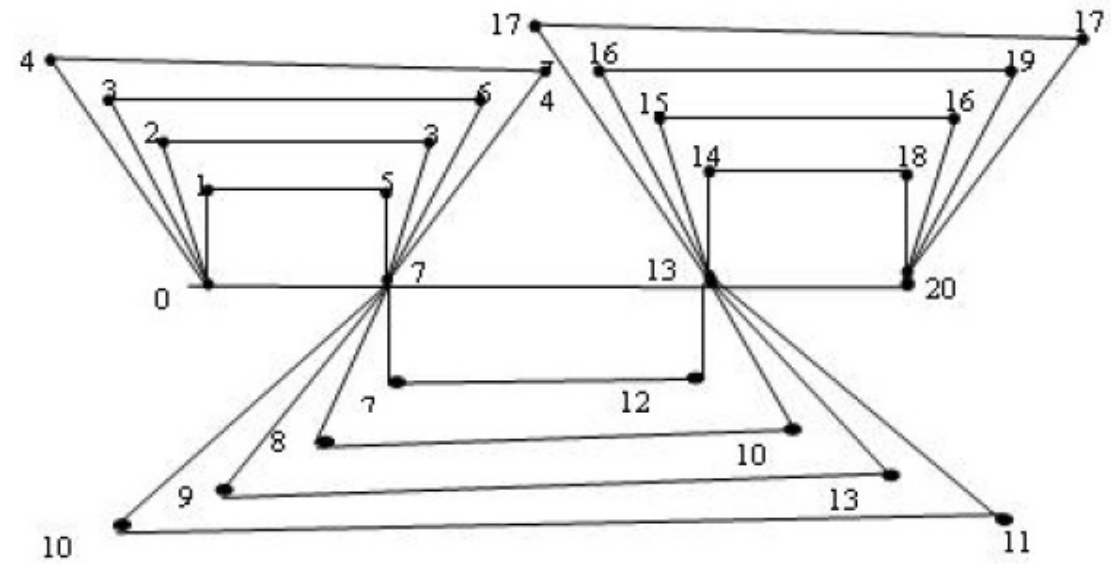

Figure 4 
Corollary 2.6. The book graph $K_{1, n} \times P_{2}$ is a vertex equitable graph.

\section{References}

[1] F. Harary, Graph Theory, Addison Wesley, Massachusetts, (1972).

[2] J. A. Gallian, A dynamic survey of graph labeling, The Electronic Journal of Combinatorics, pp. 1-389, \#DS6, (2015).

[3] A. Lourdusamy and M. Seenivasan, Vertex equitable labeling of graphs, Journal of Discrete Mathematical Sciences and Cryptography, 11 (6), pp. 727-735, (2008).

[4] P. Jeyanthi and A. Maheswari, Some Results on Vertex Equitable Labeling, Open Journal of Discrete Mathematics, 2, pp. 51-57, (2012).

[5] P. Jeyanthi and A. Maheswari, Vertex equitable labeling of cycle and path related graphs, Utilitas Mathematica, 98, pp. 215-226, (2015).

[6] P. Jeyanthi and A. Maheswari, Vertex equitable labeling of Transformed Trees, Journal of Algorithms and Computation, 44, pp. 9-20, (2013).

[7] P. Jeyanthi and A. Maheswari, Vertex equitable labeling of cyclic snakes and bistar graphs, Journal of Scientific Research, 6 (1), pp. 79-85, (2014).

[8] P. Jeyanthi A. Maheswari and M. Vijaya Laksmi, Vertex equitable labeling of double alternate snake graphs, Journal of Algorithms and Computation, 46, pp. 27-34, (2015).

[9] P. Jeyanthi A. Maheswari, and M. Vijaya Laksmi, New results on vertex equitable labeling, Journal of Algebra Combinatorics Discrete Structures and Applications, 3(2), 97-104, (2016).

[10] P. Jeyanthi A. Maheswari, and M. Vijaya Laksmi, Vertex Equitable Labeling of Super Subdivision Graphs, Scientific International, 27 (4), pp. 1-3, (2015).

[11] S.C. Shee and Y.S. Ho, The Cordiality of Path-union of $n$ Copies of a Graph, Discrete Math., 151, pp. 221-229, (1996). 


\author{
P. Jeyanthi \\ Research Centre \\ Department of Mathematics \\ Govindammal Aditanar College for Women \\ Tiruchendur-628 215, Tamilnadu, \\ India \\ e-mail : jeyajeyanthi@rediffmail.com
}

A. Maheswari

Department of Mathematics

Kamaraj College of Engineering and Technology

Virudhunagar, Tamilnadu,

India

e-mail : bala_nithin@yahoo.co.in

and

\title{
M. Vijayalakshmi
}

Department of Mathematics

Dr. G. U. Pope College of Engineering

Sawyerpuram, Tamilnadu,

India

e-mail : viji_mac@rediffmail.com 